\title{
Controlling an active bimorph deformable mirror with sub- nanometre resolution
}

loana-Theodora Nistea, Simon Alcock, Vivek Badami, Riccardo Signorato, Kawal Sawhney

loana-Theodora Nistea, Simon G. Alcock, Vivek Badami, Riccardo Signorato, Kawal Sawhney, "Controlling an active bimorph deformable mirror with subnanometre resolution," Proc. SPIE 11109, Advances in Metrology for X-Ray and EUV Optics VIII, 111090E (9 September 2019); doi: 10.1117/12.2529322

SPIE Event: SPIE Optical Engineering + Applications, 2019, San Diego, California, United States 


\title{
Controlling an active bimorph deformable mirror with sub-nanometre resolution
}

\author{
Ioana-Theodora Nistea*a, Simon G. Alcock ${ }^{\mathrm{a}}$, Vivek Badami ${ }^{\mathrm{b}}$, Riccardo Signorato ${ }^{\mathrm{c}}$, Kawal Sawhney ${ }^{\mathrm{a}}$ \\ ${ }^{a}$ Optics \& Metrology group, Diamond Light Source Ltd, Harwell Science and Innovation Campus, \\ Oxfordshire, OX11 0DE, United Kingdom; ' 'Zygo Corporation, Middlefield, CT 06455, USA; ' S.RI. \\ Tech, Italy.
}

\begin{abstract}
There is growing interest at synchrotron light and X-ray free electron laser facilities to explore and improve the dynamic performance of piezoelectric bimorph deformable X-ray mirrors. Many beamlines, especially those dedicated to Macromolecular Crystallography, need to measure hundreds of samples per day. Shorter acquisition time requires rapid changes in the focus of the X-ray beam to condense the maximum photon density onto the sample. This is necessary to match the X-ray beam to the dimensions of the sample, or to probe variable sized regions of larger samples. Fine control of the X-ray beam becomes crucial for ensuring the highest quality of scientific data and increased throughput. Previous work at Diamond Light Source successfully changed the X-ray beam focus and stabilised it in under 10 seconds using piezoelectric bimorph deformable mirrors. Further updates to the controls software of the programmable HV-ADAPTOS high-voltage power supply (from CAEN / S.RI. Tech) now make it possible to control individual electrodes at $1 \mathrm{~Hz}$ using custom voltage profiles. This allows localized compensation of piezo creep, thus improving X-ray beam shape, significantly reducing stabilisation time, and eliminating curvature drift. For ex-situ validation, dynamic changes in the surface of the bimorph mirror need to monitored in real-time with sufficient spatial sensitivity. In this paper, we show that the active optical surface of a bimorph mirror (from Thales-SESO) can be accurately changed with sub-nanometre height sensitivity by dynamically monitoring the mirror's surface using an array of high-speed (up to $200 \mathrm{kHz}$ ) Zygo ZPS $^{\mathrm{TM}}$ absolute interferometric displacement sensors mounted in an independent metrology frame.
\end{abstract}

Keywords: optical metrology, displacement interferometry, bimorph X-ray mirrors, active optics, absolute fiber interferometer

*ioana.nistea@diamond.ac.uk

\section{INTRODUCTION}

Diamond Light Source (Diamond) ${ }^{\mathbf{1}}$ is the UK's national facility for generating multiple, ultra-intense beams of synchrotron radiation. These photon beams, predominantly X-rays, are simultaneously utilized by several experimental stations (so-called beamlines). This enables research in a broad spectrum of scientific and technological fields: from viruses and vaccines, to fossils and jet engines. Since experiments began in 2007, many thousands of researchers from academia and industry have used Diamond's beamlines to perform scientific research, assisted by over 620 scientific, technical, and administrative staff. Currently, 33 beamlines are operational at Diamond, many of which have been significantly upgraded since operations began 12 years ago.

The successful exploitation of the intense synchrotron light depends to a significant extent on the quality and performance of beamline optics. Each beamline relies on a dedicated set of optics to monochromate, shape and focus the $\mathrm{X}$-ray beam to their needs. The Optics and Metrology (O\&M) group contributes to every aspect of designing, procuring, testing, and installing these beamline optics. To further extend the capabilities of Diamond's beamlines, and continue to achieve world-leading performance, the O\&M group is also actively pursuing a broad portfolio of targeted research activities, including development of next-generation optics, instrumentation, and techniques. This is supplemented by active collaborations with key external research institutes and specialized industries.

Each beamline specializes in several experimental techniques, including crystallography, diffraction, spectroscopy, tomography, magnetic materials, ptychography, absorption, imaging, microscopy, and scattering. This dictates that the optical layout needs to be quickly and efficiently reconfigurable to tune the size, shape, and energy of the X-ray beam. 
To achieve this, certain optics can be inserted into, or retracted from, the X-ray beam. Most beamlines also contain one or more actively bent mirrors, either using mechanical or piezoelectric actuators.

Piezo-electric "bimorph" deformable mirrors ${ }^{2}$, manufactured by Thales-SESO (France), are used to focus X-rays on several beamlines at Diamond, and also at many other facilities around the world. However, soon after scientific operations began at Diamond in 2007, several beamlines using bimorph mirrors reported problems with the size and shape of their X-ray beam. The O\&M group coordinated an extensive internal investigation to understand the causes of these problems, and to work with suppliers to find a solution ${ }^{3}$. Re-polishing the older $1^{\text {st }}$ generation bimorph mirrors at Thales-SESO removed the "junction effect", led to considerable improvements in X-ray performance and extended lifetime. Subsequently, $2^{\text {nd }}$ generation bimorph mirrors were developed at Thales-SESO ${ }^{3}$, which were designed not to suffer from the junction effect. Since then, over many years, we have continued to collaborate with specialized companies to create novel bimorph mirrors and mounting methods. In addition to hardware improvements, we have also investigated how to utilize bimorphs to achieve the full extent of their capabilities ${ }^{5-9}$.

Currently, the tangential curvature of bimorph mirrors is typically only changed every few hours or even days. This operation can take tens of minutes and often requires expert guidance or use of pre-established look-up tables. Following a large change in curvature, bimorph mirrors are known to take > 15 minutes to stabilize on the nanometer scale, although this often had a negligible impact on beamline performance. Therefore, historically, the dynamic performance of active X-ray optics has not been fully exploited at synchrotron and X-ray free electron laser (XFEL) facilities. Several of the intrinsic capabilities of the mirror hardware remained largely underutilized. This is in stark contrast to ultra-fast adjustments made to adaptive optics in other scientific fields such as Astronomy. However, many synchrotron beamlines, especially those dedicated to Macromolecular Crystallography, now analyse hundreds of samples per day. Such beamlines would greatly benefit from the ability to rapidly modify the X-ray beam profile in only a few seconds to match to the size of smaller samples, or to vary the illuminated region of larger samples, or to control the X-ray photon flux density. Furthermore, as the synchrotron and XFEL user community becomes more diverse, and perhaps are not trained in the detailed operation of a beamline, a key goal is for the user to "dial in" the desired X-ray beam parameters without the intervention of skilled personnel.

In an earlier study ${ }^{\mathbf{1 0 , 1 1}}$, we investigated the time-dependent behavior of a series of micro-focus, bimorph mirrors using Fizeau interferometry in the Optical Metrology Lab (OML) at Diamond. A novel "speedy" bimorph was developed by making several hardware and software improvements. Utilising a new high-voltage power supply, the magnitude of the mirror's curvature drift after applying a very large voltage shift to the piezo actuators was significantly reduced. The residual drift, attributed to piezoelectric creep, was shown to be repeatable and could readily be compensated. Once installed on the Microfocus Macromolecular Crystallography (I24) beamline at Diamond, the KB pair of bimorphs focused the X-ray beam in < 10 seconds. Of equal importance, compensating piezo creep ensured that the size of the Xray beam remained stable for more than one hour after making a major change. This enables continuous "adaptive" shaping of the X-ray beam in almost real time. Such innovations could lead to significant scientific improvements in how active / adaptive X-ray optics are utilized at synchrotron and XFEL sources.

The limiting factor for ex-situ metrology was the acquisition speed of the MiniFiz150 Fizeau interferometer, which could only obtain a high-quality image of the optical surface every 10 seconds. To extend this investigation we collaborated with Zygo Corporation (USA) to utilize their high-speed ZPS ${ }^{\mathrm{TM}}$ displacement sensors to dynamically monitor a bimorph mirror at $\mathrm{kHz}$ frequencies whilst voltages were applied to deform the optical surface ${ }^{\mathbf{1 2}}$.

\section{EXPERIMENTAL}

\subsection{Optical Metrology Lab (OML)}

The OML is a class 10,000 (ISO 7) cleanroom with a floor area of $\sim 100 \mathrm{~m}^{2}$. The OML contains a suite of metrology instruments ${ }^{13}$, including the Diamond-NOM ${ }^{14}$, which are capable of resolving ultra-small optical defects on the nanoscale. Prior to beamline installation, all X-ray optics for Diamond's beamlines are routinely characterized and optimized in their opto-mechanical holders in the OML. The environment inside the OML is actively controlled to achieve temperature stability of $\sim 15 \mathrm{mK}$ over any 24 hour period. Dedicated enclosures around each metrology instrument further enhance this stability. 


\subsection{Piezo-electric bimorph deformable mirror and its opto-mechanical holder}

The optic used in this study is a $2^{\text {nd }}$ generation piezo-electric bimorph deformable mirror (see Figure 1). It was manufactured by Thales-SESO (France) and is representative of many such mirrors used at different beamlines around the world. The single-crystal silicon substrate is $600 \mathrm{~mm}$ long and contains 16 independent piezo-electric actuators. Heavy metal coatings of $\mathrm{Rh}$ and $\mathrm{Pt}$ deposited on the substrate provide enhanced reflectivity over a large X-ray wavelength range. Further details about this mirror are provided in ${ }^{4}$. A custom-built opto-mechanical holder for this mirror was designed and built by Cinel (Italy). Metallic, spring-loaded clamps securely hold the mirror, without overconstraining it. This versatile holder is designed to be reconfigurable such that it can hold the mirror with its surface normal pointing upwards, downwards, or sideways. This mirror has been used on several beamlines at Diamond whilst their old $1^{\text {st }}$ generation bimorphs were shipped back to Thales-SESO for re-polishing.

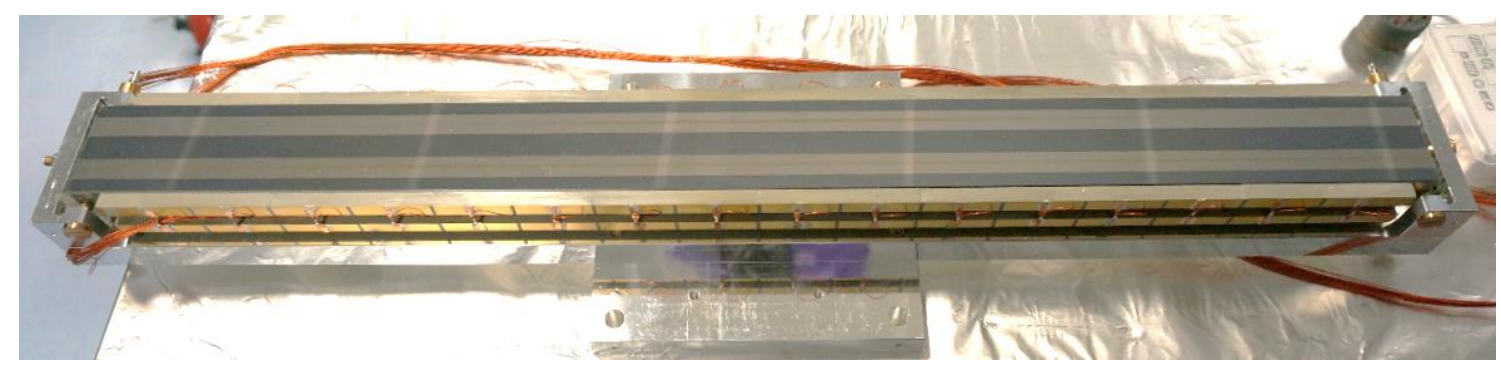

Figure 1 . The $2^{\text {nd }}$ generation piezoelectric bimorph deformable mirror used in this study, mounted in its custom-built optomechanical holder.

\subsection{High-voltage power supply}

Voltages were supplied to the bimorph mirror using an $\mathrm{HV}^{-A D A P T O S}{ }^{15}$ bipolar high-voltage power supply, which is manufactured by CAEN (Italy) and is then customized and distributed by S.RI. Tech (Italy). This model (see Figure 2) is used to control $\sim 20$ bimorphs on 5 beamlines at Diamond. For this project, our 16 channel HV-ADAPTOS was reprogrammed to output unique voltages to the individual electrodes of the bimorph mirror, with a resolution of $0.1 \mathrm{~V}$, at a refresh rate of $1 \mathrm{~Hz}$. This upgrade enables high-speed control of localized features on the mirror.

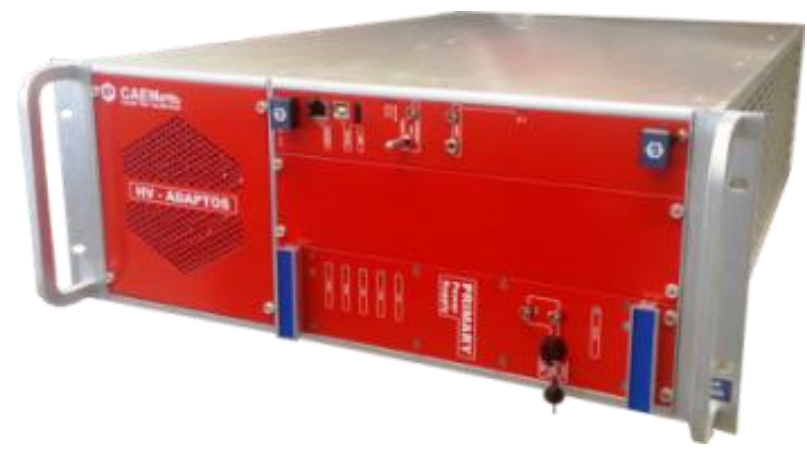

Figure 2. The HV-ADAPTOS bipolar high-voltage power supply applies voltages to the multiple electrodes of each bimorph mirror.

\subsection{ZPS multi-sensor, interferometric system}

The ZYGO ZPSTM is an absolute fiber interferometer system with a multiplicity of channels designed for applications which require tens of measurement channels operating simultaneously $\mathbf{1 6}_{\mathbf{1 7}, 18,19}$. Examples of such applications are real- 
time monitoring of the shape of deformable structures and form measurement of complex shapes. The system employs miniature, fiber-based, probes to interferometrically measure the absolute position of a target relative to the reference surface of the sensor (see Figure 3). This system distinguishes itself from other interferometric systems in that it measures the absolute distance to the target, and not just the displacement from an arbitrary zero position. Table 1 summarizes the key specifications of the ZPS system.

Table 1. Key specifications of the ZYGO ZPS absolute interferometer.

\begin{tabular}{|l|l|}
\hline \multicolumn{1}{|c|}{ Parameter } & \multicolumn{1}{c|}{ Value } \\
\hline Size of sensors & $27 \mathrm{~mm}$ long, 3mm diameter \\
\hline Number of channels & Up to 64 sensors \\
\hline Working distance from target & $3.5 \mathrm{~mm}$ \\
\hline Measurement range & $\pm 0.6 \mathrm{~mm}$ \\
\hline Noise Density $(3 \sigma)$ & $0.02 \mathrm{~nm} \mathrm{~Hz}{ }^{-1 / 2}$ \\
\hline Stability & $<1 \mathrm{~nm} /$ day \\
\hline Absolute position repeatability $(3 \sigma)$ & $0.5 \mathrm{~nm}$ \\
\hline Non-linearity & $\pm 1 \mathrm{~nm}$ \\
\hline Acquisition rate & $\mathrm{Up} \mathrm{to} 208 \mathrm{kHz}$ \\
\hline Vacuum compatibility & $<10^{-9} \mathrm{Torr}$ \\
\hline
\end{tabular}
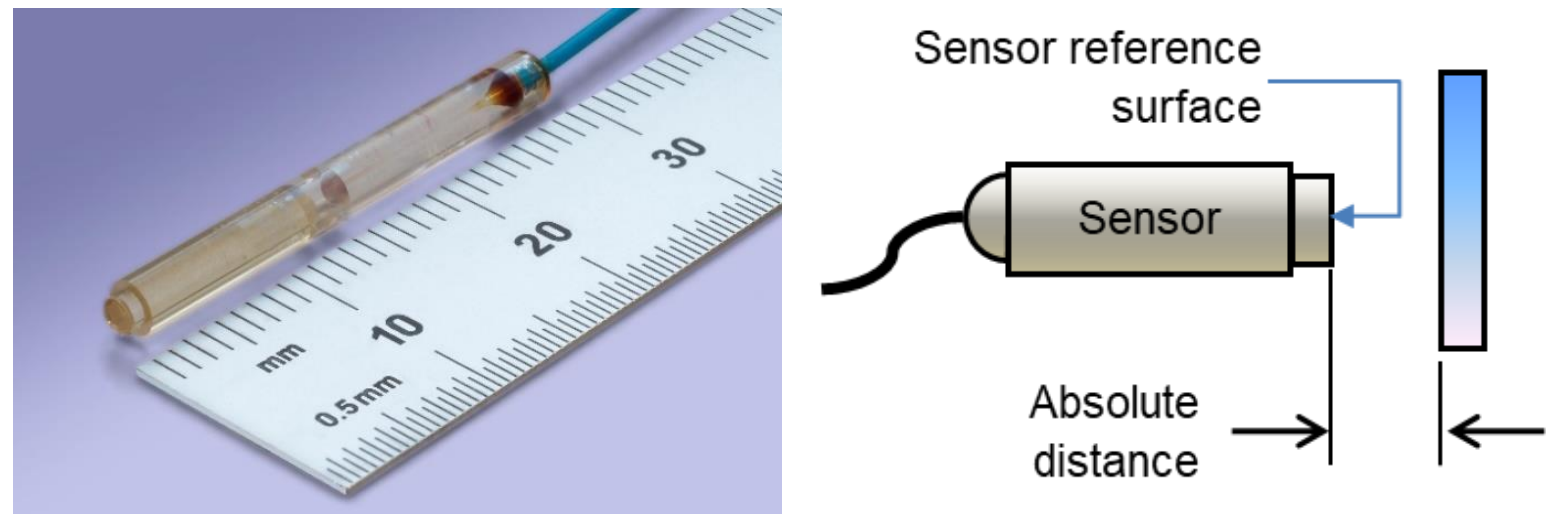

Figure 3. (a) A ZPS sensor (b) Distance measurement principle of each interferometric ZPS sensor.

\subsection{ZPS system mounted on the bimorph mirror}

Figure 4 illustrates the basic measurement principle, wherein an array of ZPS sensors, oriented with their optical axes nominally perpendicular to the mirror, continuously monitor the optical surface and any changes thereof. A very stable metrology frame, independent from the bimorph mirror's opto-mechanical holder, contains the sensor array, and incorporates a cutout to enable simultaneous measurement of the mirror's surface by an additional metrology instrument such as the Diamond-NOM slope profilometer or a Fizeau interferometer. Two rows of ZPS sensors straddle the cutout such that the pairwise average of the sensors establishes an effective line of measurement along the center of the cutout. This enables comparison with other measurement methods in compliance with the Abbé principle. The experimental apparatus consists of two main parts: (i) the bimorph mirror and its holder; (ii) a custom-built metrology frame and bipod 
supports for the ZPS probes. A manually adjustable stage for tip / tilt / z, mounted to an interface plate, supports the bimorph mirror's holder. This allows manipulation of the mirror in three rigid-body degrees-of-freedom (DOF): rotations about the $\mathrm{x}$ and $\mathrm{y}$ axes to orient the mirror within the angular acceptance of ZPS sensors; and translation in $\mathrm{z}$ to set the mirror's surface height at the optimal stand-off distance of $3.5 \mathrm{~mm}$. The metrology frame which holds the ZPS sensors also mounts to the interface plate via three bipods which constrain the metrology frame kinematically. The bipods prevent distortion in the shape of the frame due to disturbance forces that can result from several effects such as: differential expansion between the interface plate and the frame; and transmission of parasitic deflections of the mirror holder to the metrology frame from the mirror deformation. In conjunction with a high conductivity material for the frame, which avoids distortion due to thermal gradients, sub-nanometer stability in the shape of the frame is achievable with the prevailing temperature stability of the environment in which these tests were conducted. The bipod / frame assembly has a first resonance of $200 \mathrm{~Hz}$ to minimize the effects of acoustic and seismic vibrations from the environment. The frame contains two rows of 19 sensors on either side of the central cutout in the metrology frame (Figure 4). While the sensor spacing of $\sim 23 \mathrm{~mm}$ was adequate for the comparison of low-order deformations in this study, this is not a fundamental limitation. The sensors could potentially be spaced only a few $\mathrm{mm}$ apart to enable characterization of higher spatial frequency changes to monitor more complex mirror shape changes. Figure 5a shows the bimorph mirror in its opto-mechanical holder, while Figure 5b shows the ZPS metrology frame and the associated hardware. Figure $5 \mathrm{c}$ shows the two components as they are assembled together.

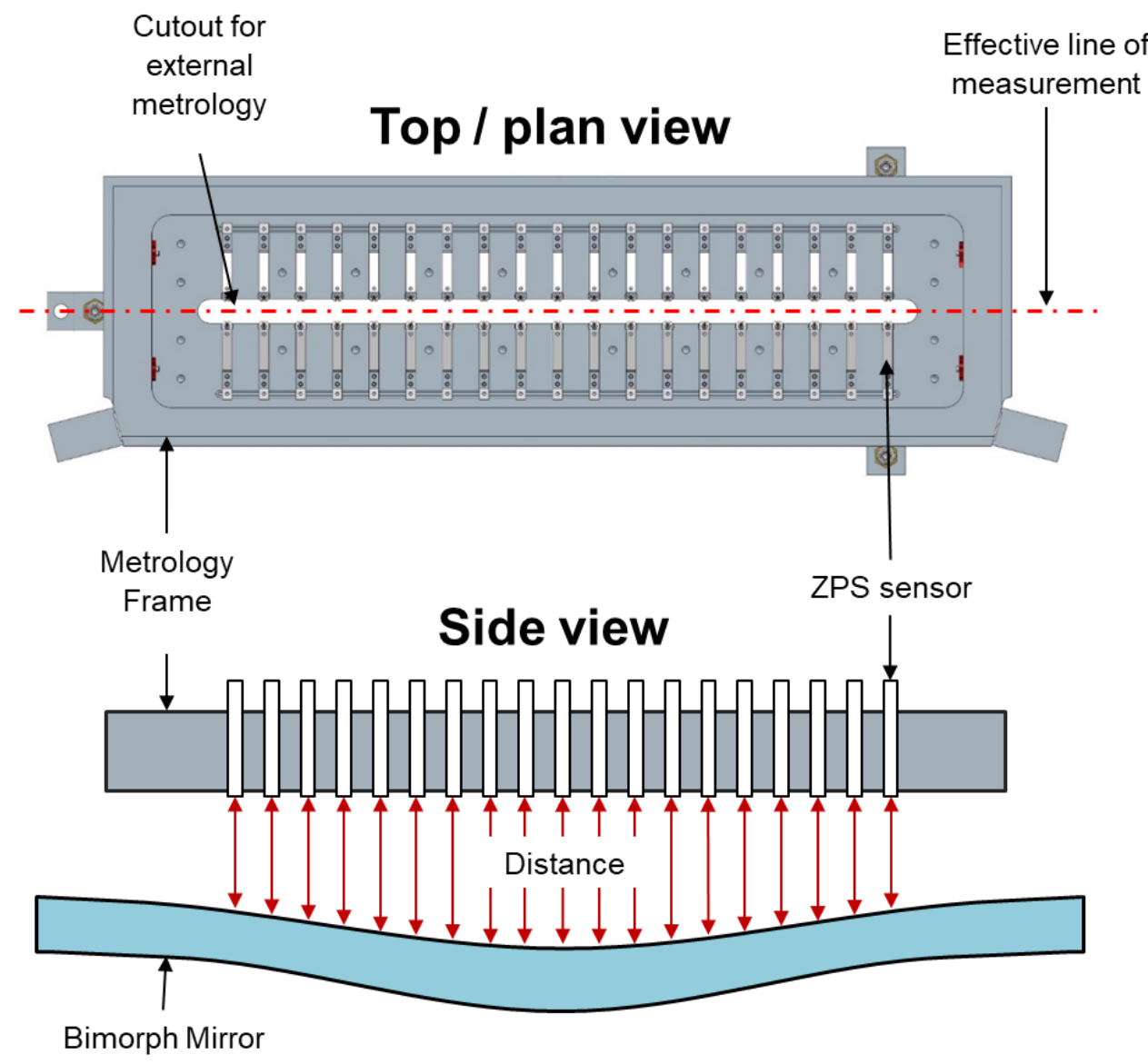

Figure 4. ZPS sensors mounted in a custom-built metrology frame are suspended $\sim 3.5 \mathrm{~mm}$ above a bimorph mirror. The multiple ZPS sensors monitor any changes in the optical surface of the bimorph as it is subjected to voltage changes. 

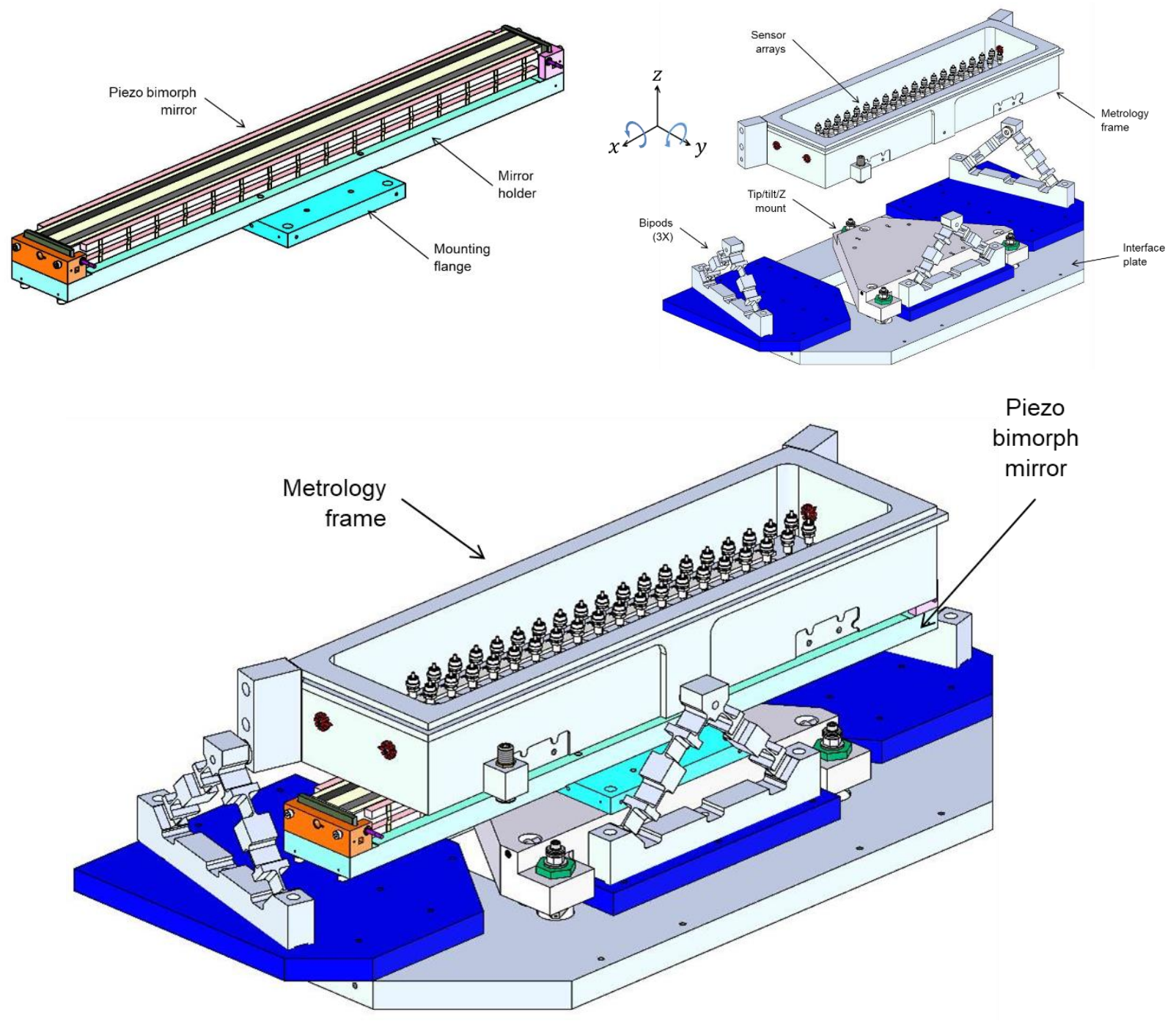

Figure 5. Details of experimental setup: (a) Piezoelectric bimorph deformable X-ray mirror in its custom-built optomechanical holder; (b) Metrology frame for ZPS sensors and tip / tilt / z stage; and (c) full setup, with mirror installed.

\subsection{Optical metrology using the Diamond-NOM and ZPS system}

The bimorph mirror and ZPS system were installed on the Diamond-NOM in the OML (Figure 6). The NOM is contained inside a passively controlled, ultra-stable environment. The air temperature, pressure, and humidity are logged by a Vaisala weather station every $\sim 4$ seconds. A refractometer, which directly measures the refractive index of air in close vicinity to the experiment, is used to compensate the ZPS probes for environmental disturbances. For each Diamond-NOM scan, the mirror's height profile was sampled at $1 \mathrm{~mm}$ intervals along the central, tangential line of the mirror (440 points). Correspondingly, the 19 ZPS sensors in each row sampled along the 2 coated stripes of the mirror at $\sim 23 \mathrm{~mm}$ intervals. Table 2 provides a comparison of the experimental parameters of the two metrology systems operating in parallel. 
Table 2. Comparison between the two metrology instruments used to monitor the optical surface of the bimorph mirror

\begin{tabular}{|l|l|l|}
\hline & Diamond-NOM & ZPS system \\
\hline Measurand & Slope & Distance / height \\
\hline Type of scan & 1 D scans & 2 rows of sensors \\
\hline Measurement duration & $\sim 26$ min for each scan & Simultaneous @ 10 's of kHz \\
\hline Lateral sampling on mirror & $1 \mathrm{~mm}$ & $23 \mathrm{~mm}$ \\
\hline Number of measurement points & $440(1$ point per $\mathrm{mm})$ & $38(19$ in each row $)$ \\
\hline
\end{tabular}

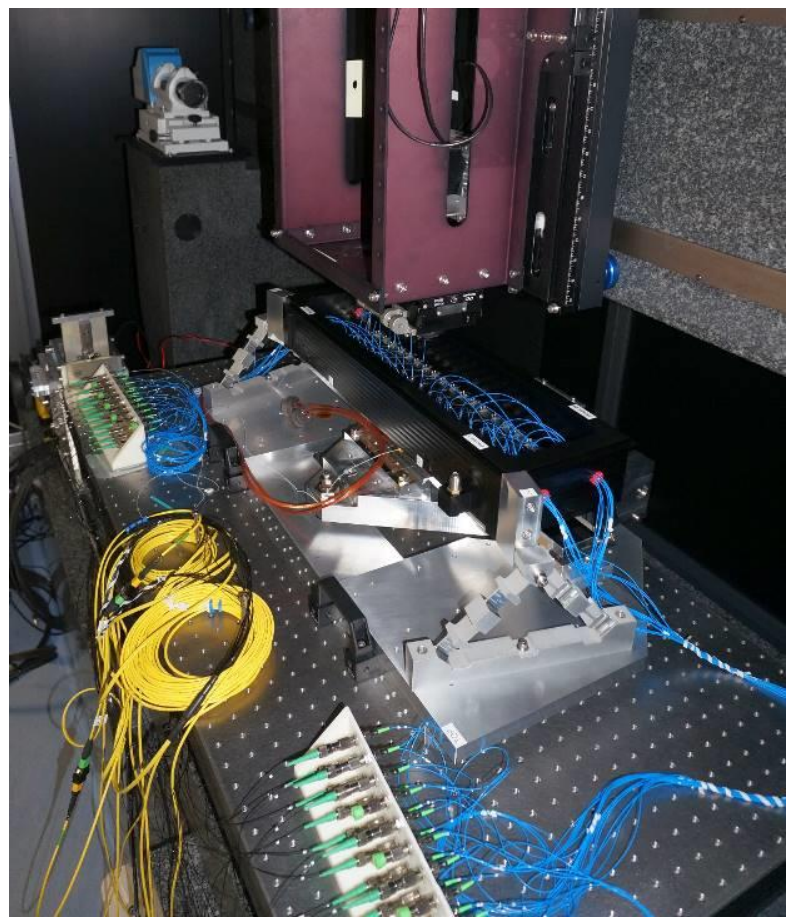

Figure 6. Bimorph mirror and ZPS sensor frame installed on the Diamond-NOM slope profilometer

\section{RESULTS}

\subsection{Bending range and stability}

The first sets of tests simultaneously measured the global bending of the bimorph mirror, using both the Diamond-NOM and the ZPS probes. Best fit $2^{\text {nd }}$ order polynomials were calculated for each measurement of the mirror's height profile to extract the dynamic cylindrical / spherical radius of curvature as a function of time. The inverse of the radius (so-called "curvature") follows a well-known linear relationship with applied voltage for bimorph mirrors. Bending of the mirror was achieved by applying incremental steps of $\pm 500 \mathrm{~V}$ each hour between a minimum voltage of $-1000 \mathrm{~V}$ and a maximum of $+1000 \mathrm{~V}$. Two such cycles of bending are shown in Figure $7 \mathrm{a}$ which reveals that the "curvature" data (1 / radius) acquired by the Diamond-NOM (blue circles) is in good absolute agreement with the ZPS sensors (red dots, which form a semi-continuous line) over the full bending range of the bimorph mirror. This reassuringly provides confidence that the measurement principle is fit for purpose. Each Diamond-NOM scan was collected over a 26 minute period, whereas the ZPS probes acquired data every 10 seconds. In effect, each NOM scans records an average of the mirror's curvature over the acquisition period, highlighting the intrinsic limitations of using this instrument for faster studies of the dynamics of optical surfaces. The higher-speed ZPS data also reveals the settling of the mirror's shape 
after each voltage jump. Longer term stability tests, shown in Figure 7b, were performed to characterize how the mirror's curvature drifted over many hours after making the largest possible voltage changes that the mirror can safely accommodate ( 0 to -1000 to $+1000 \mathrm{~V}$ and back). This corresponds to a cylindrical radius change from 1.3 to $>4 \mathrm{~km}$. As before, blue circles represent the inverse radius of curvature as measured by the Diamond-NOM, and the red dots represent the same parameter measured using the ZPS system. Once again, the excellent agreement confirms that the apparatus and environment are suitably stable, and both metrology instruments are capable of accurately measuring the long-term drifts in the bimorph's optical profile.
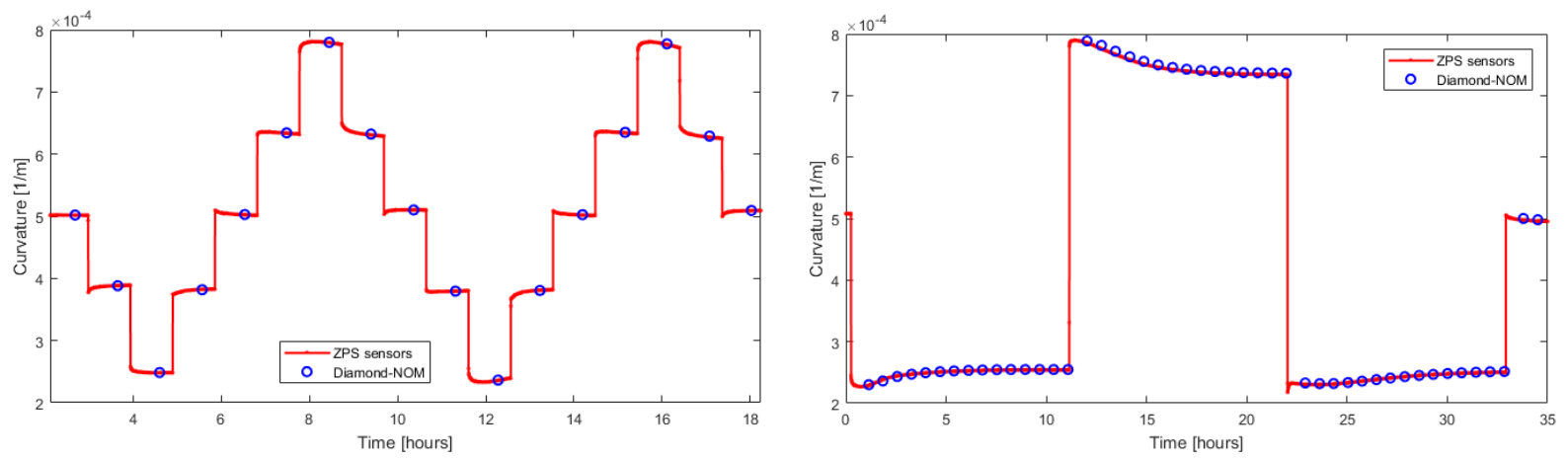

Figure 7 Curvature (inverse of radius) of the bimorph mirror, as measured by the Diamond-NOM (blue circles) and ZPS sensors (red lines), measuring how the mirror bends in (a) $\pm 500 \mathrm{~V}$ increments over its full range, and (b) longer term stability after applying the maximum change of $\pm 2000 \mathrm{~V}$. Good agreement is observed between both instruments.

\subsection{Piezo response functions of the bimorph mirror}

Piezo response functions (PRFs) are a simple and effective method to characterize the change in the bimorph mirror's height profile in response to a fixed voltage being sequentially applied to each individual piezo actuator. Using the measured PRFs, inverse matrix methods are used to calculate the voltage required for every piezo electrode to cumulatively bend the mirror to any given shape (within limitations of the hardware). The PRFs were measured for this mirror by sequentially applying steps of $400 \mathrm{~V}$. Figure $8 \mathrm{a}$ shows the PRFs obtained by the Diamond-NOM, and Figure $8 \mathrm{~b}$ shows the PRFs as measured by the ZPS system. Note that each PRF curve is normalized to show a change in the mirror's height profile per volt applied, and that only the response of the central 12 piezo actuators was recorded due to the length of the aperture in the ZPS metrology frame. The output from each of the 19 ZPS sensor in a single row is shown by a discrete point in Figure 8b. By fitting a smooth function to the displacement values from the ZPS sensors we obtain continuous PRF curves. Data from both metrology instruments very closely match each other and show that the mirror's surface can be controlled on the sub-nanometer level.
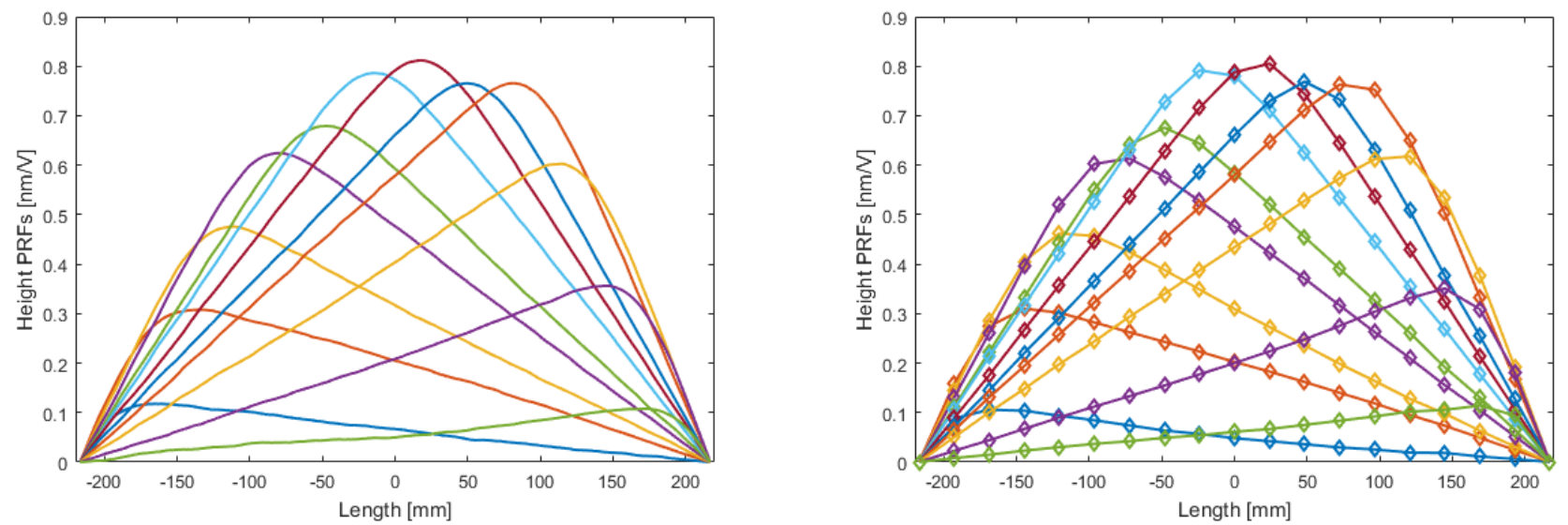

Figure 8. Piezo response functions (PRF) of the 12 central piezo actuators of the bimorph mirror as measured by (a) the Diamond-NOM and (b) the ZPS sensors. Each discrete point shows the output of an individual ZPS sensor. 


\subsection{Resolving nanometer-sized changes in the mirror's sagitta}

The next test was to investigate whether extremely small changes in the mirror's shape could be observed in response to applying voltage changes to the mirror of $\leq 5 \mathrm{~V}$. Figure 9a and Figure 9b show the change in the mirror's shape as small steps of $0.5,1,2$, or $5 \mathrm{~V}$ were applied to all piezo electrodes, as measured non-simultaneously by the Diamond-NOM and the ZPS probes respectively. The Diamond-NOM clearly resolves changes induced by 2 and $5 \mathrm{~V}$ steps, but struggles to discern $<1 \mathrm{~V}$ voltage steps. However, the ZPS can reliably and repeatably measure even $0.5 \mathrm{~V}$ changes, corresponding to $<3 \mathrm{~nm}$ changes in the mirror's sagitta (the depth at the center of mirror, compared to its two ends). This result shouldn't be misunderstood as an absolute limitation of the Diamond-NOM for measuring at the nanometer level, but simply a consequence of the way it acquires data in a step-by-step fashion along the mirror's profile for a duration of 26 minutes. During this time, the very small curvature change has enough time to drift and become unrecognizable. The Diamond-NOM is proven to be capable of delivering sub-nanometer resolution and repeatability by averaging multiple scans, but the measured surface needs to be extremely stable throughout. The bimorph mirror in this particular test is a 'moving target', in that the small change in its shape are comparable to the typical curvature drift due to piezoelectric creep during the time it takes the NOM to complete a scan. The ZPS sensors measure the entire surface simultaneously in a fraction of a second. In this manner, the instrument captures a snapshot of the mirror's figure change immediately after applying the voltage change. This makes it an invaluable tool for monitoring fast, dynamic phenomena.
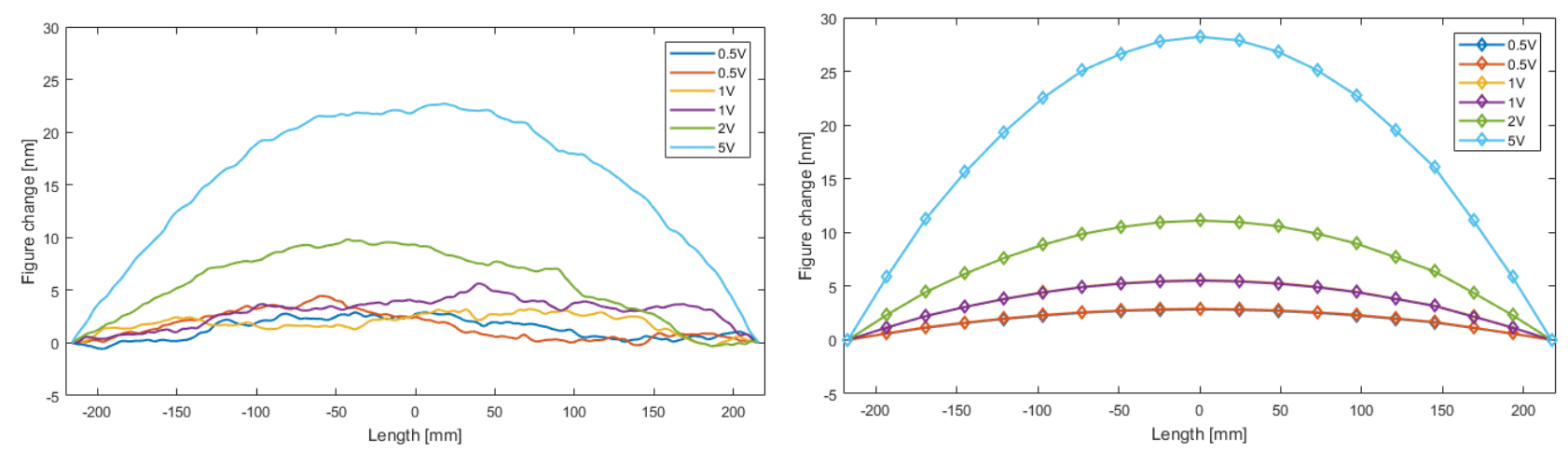

Figure 9. Hight change in the mirror's surface for voltage steps between 0.5 and $5 \mathrm{~V}$, as measured by (a) the Diamond-NOM and (b) the ZPS sensors. The ZPS probes can easily resolve changes of a few nanometers in the mirror's surface induced by only $0.5 \mathrm{~V}$ pulses.

Having confirmed that the ZPS system can resolve $<5 \mathrm{~nm}$ changes in the mirror's sagitta, induced by only $0.5 \mathrm{~V}$ changes to the piezo electrodes, we proceeded to find its ultimate limit. We therefore applied even smaller increases of only $0.1,0.2,0.3$, or $0.5 \mathrm{~V}$ to all piezos and monitored the mirror using the ZPS probes. Note that $0.1 \mathrm{~V}$ is the smallest voltage increment that that the power supply can achieve. Figure 10 shows the change in the mirror's profile as measured by the ZPS sensors. Amazingly, the ZPS system can comfortably measures changes of 500 picometres in the mirror's sagitta, corresponding to a cylindrical radius change of $47000 \mathrm{~km}$ ! To put this into perspective, this is more than 7 times greater than the radius of the Earth! Note that there were two steps of $0.1 \mathrm{~V}$, and two steps of $0.2 \mathrm{~V}$, and although there are small differences between each group, this imperfect repeatability amounts to a peak-to-valley of $<100$ picometres! 


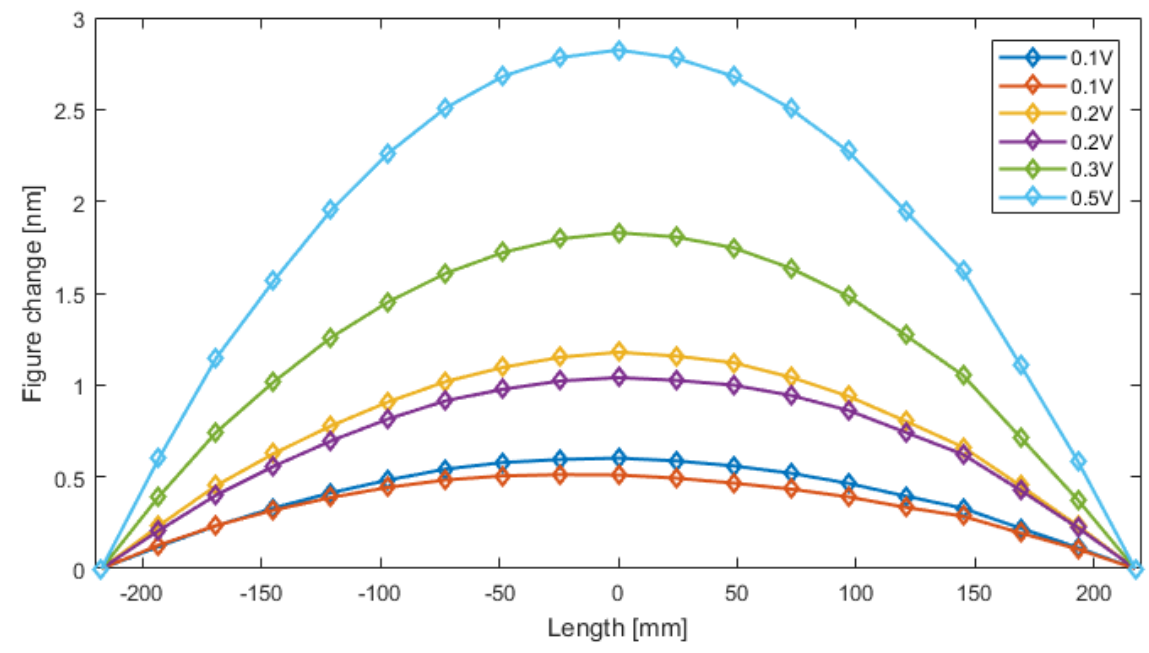

Figure 10. Change in the mirror's surface induced by voltage steps of 0.1 to $0.5 \mathrm{~V}$. For the smallest voltage changes of 0.1 $\mathrm{V}$, the ZPS system is shown to be capable of reliably measuring a 500 picometre change in the mirror's sagitta, corresponding to a radius change of $47000 \mathrm{~km}$ !

\subsection{High-speed capabilities of the ZPS probes}

The previous tests have already hinted at the excellent dynamic capabilities of the ZPS system. To investigate further, we use the ZPS sensors at a faster acquisition rate of $10 \mathrm{kHz}$ to monitor the bimorph mirror's shape whilst steps of $\pm 0.1 \mathrm{~V}$ were applied to all piezo electrodes every 5 seconds. The displacement data from the ZPS probes was batch processed in MATLAB ${ }^{\circledR}$ to automatically calculate the sagitta of the mirror as a function of time. As seen in Figure 11, and confirming the results in Figure 10, the mirror's sagitta repeatedly changed by 500 picometres for each $0.1 \mathrm{~V}$ change. Interestingly, the ultra-high sensitivity of the ZPS sensors enabled damped oscillations in the mirror's shape of $<100$ picometres amplitude to be observed immediately after each voltage step. In effect, the mirror is undergoing a damped "ringing effect" after each voltage impulse is received! Very importantly, the data was collected in "open loop" mode: the ZPS system was simply monitoring the mirror's shape, and no voltage feedback was being applied after each $0.1 \mathrm{~V}$ step. The level of accuracy and backlash-free behavior is an intrinsic property of the complete system (i.e. mirror plus holder plus power supply).

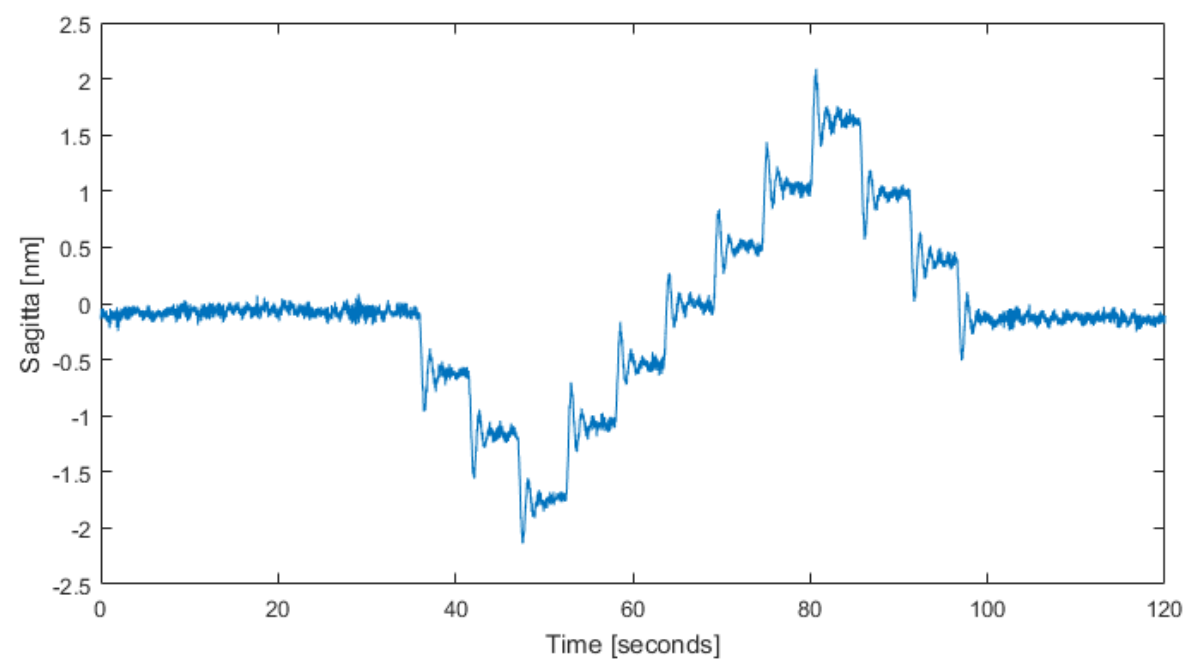

Figure 11. Temporal evolution of the mirror's sagitta (depth) acquired by ZPS sensors at $10 \mathrm{kHz}$ whilst successive voltage steps of $\pm 0.1 \mathrm{~V}$ were applied to the bimorph. Features $<100$ picometres in height can be readily resolved. 


\subsection{Correcting distortions in the $\mathrm{X}$-ray wavefront}

Having investigated the temporal and spatial sensitivities of the ZPS sensors, we progressed to using them to monitor changes in a bimorph mirror that would have beneficial effects for an X-ray beamline. Aside from thermal insensitivity, speed, and over-heating / fatigue issues during continuous operation, the main advantage of bimorph mirrors compared to mechanically bent mirrors is the superior number of actuators. Whereas mechanical benders typically have only 1 or 2 actuators (which bend the mirror into $2^{\text {nd }}$ or $3^{\text {rd }}$ order polynomial shapes in height), bimorph mirrors generally have many more actuators (16 in this case) and can therefore achieve more complicated profiles. This enables the possibility of using bimorph mirrors to purposefully apply novel changes to the X-ray beam ${ }^{20}$. In general, the divergence distribution of the incoming X-ray beam can be manipulated to create a user-defined, outgoing X-ray wavefront to suit a range of scientific applications. For example, a bimorph mirror can readily be bent into a $4^{\text {th }}$ order polynomial shape to compensate for: deformations to the mirror caused by gravity sag; or photon-induced, thermal heat bumps on upstream optics. This later issue is often a problem with double crystal monochromators, which will likely worsen when the Diamond machine is upgraded in the coming years to achieve a lower emittance. To highlight the adaptability of bimorph mirrors, the PRF and the inverse-matrix method were used to determine the necessary voltage settings required to bend the mirror into a range of user-defined shapes including Gaussian's or Lorentzian's. Figure 12a and Figure 12b shows the measured output from the ZPS sensors as the mirror was bent into a Gaussian or a Lorentzian respectively. The movie in Figure 13 shows the output form the ZPS sensors as the mirror's profile was changed every 10 seconds between a $4^{\text {th }}$ order polynomial, Gaussian, and Lorentzian curves.
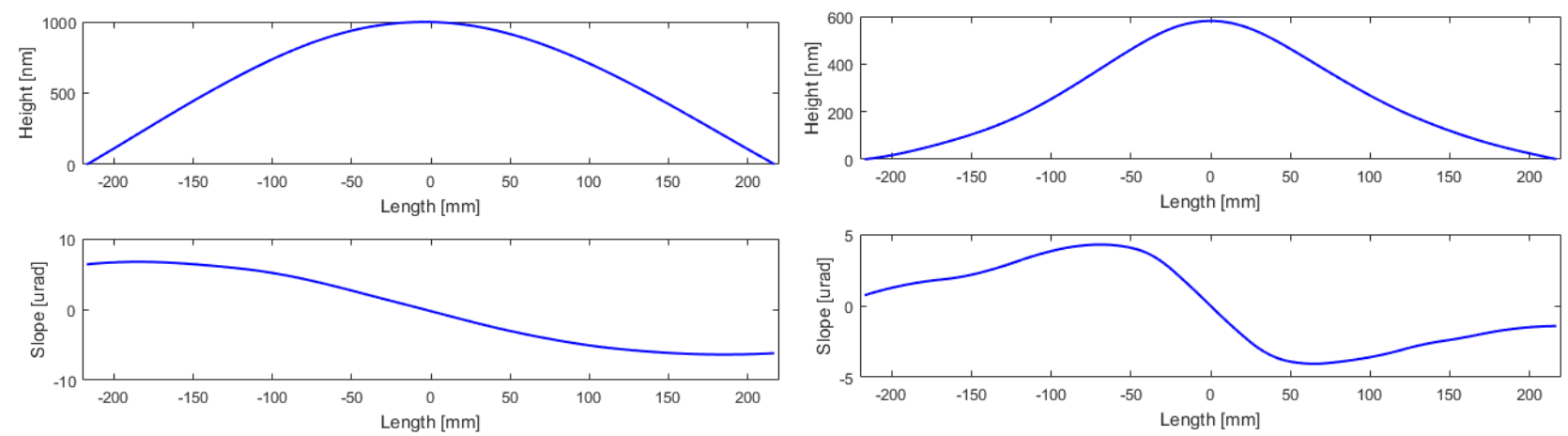

Figure 12. ZPS sensors monitoring the shape of a bimorph mirror as it was bent into complex surface profiles including (a) a Gaussian and (b) a Lorentzian. The upper charts show the height change along the mirror's length, and the lower charts show the corresponding change in slope. 

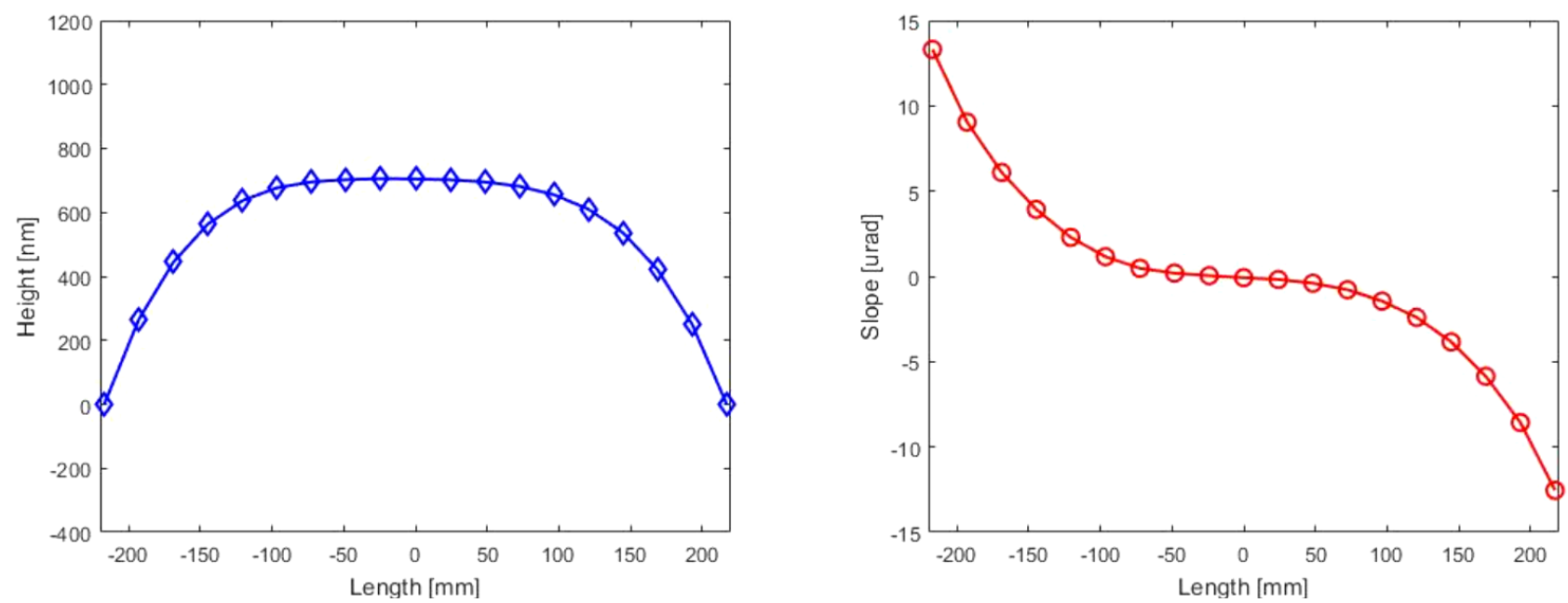

Figure 13. Video 1: Movie showing the output from the ZPS sensors as the bimorph mirror's profile was dynamically changed between higher-order shapes $\left(4^{\text {th }}\right.$ order polynomial, Gaussian and Lorentzian). http://dx.doi.org/10.1117/12.2529322.1

\section{CONCLUSIONS}

In this work we have demonstrate two important results about the high-speed use of piezoelectric bimorph deformable $\mathrm{X}$-ray mirrors. Firstly, the combination of a $2^{\text {nd }}$ generation bimorph mirror, coupled with a strain-free holder and a stateof-the-art programmable high-voltage power supply, can accurately and rapidly produce extremely small $(<500$ picometres) changes in the optical surface which reflects X-ray beams at synchrotron or free electron laser sources. Secondly, the multi-sensor ZPS interferometric system can accurately measure both large (10's of micrometres) and extremely small $(<500$ picometres) changes in the surface of an active bimorph mirror. High-speed $(\mathrm{kHz})$ simultaneous acquisition of multiple ZPS probes allows the user to capture an instantaneous snapshot of the mirror's profile, as well as dynamic changes during and after shape changes, including ringing phenomena. This information can be used to very precisely $(\ll<1 \mathrm{~nm})$ and very quickly control the surface of the bimorph. At present, this open-loop control strategy uses no feedback and operates every few seconds. This is limited only by the current controls software of the high-voltage power supply. We also verified that bimorph mirrors can purposefully be bent into a range of higher-order surface profiles, which could correct X-ray wavefront aberrations from imperfect, upstream X-ray optics, or create a range of novel shapes for the reflected X-ray beam. These preliminary results show that a combination of the ZPS sensors and a programmable HV power supply with a faster update rate could achieve closed-loop control of the mirror's profile. This makes it possible to program the shape of a bimorph mirror to achieve a range of desired profiles in a fast and repeatable fashion, whilst also compensating for the effects of thermal drift, actuator creep and photon induced deformations. This has the important potential to: i) allow a prescribed shape to be programmed by an inexperienced user without expert intervention; ii) make rapid, accurate changes to the X-ray beam size and shape; and iii) maintain the characteristics of the X-ray beam, even in the face of environmental disturbances or changes in upstream optics.

\section{ACKOWLEDGEMENTS}

The Authors would like to thank Matteo Fusco at CAEN for expertly reprogramming the controls software and the userinterface for the HV-ADAPTOS. We would also like to thank John Sutter and Andy Foster at Diamond for their continued efforts to develop software scripts to make it easier to use bimorph mirrors on Diamond's beamlines via EPICS. 


\section{REFERENCES}

[1] www.diamond.ac.uk

[2] Signorato, R., Hignette, O. and Goulon, J., "Multi-segmented piezoelectric mirrors as active/adaptive optics components,” J. Synchrotron Radiat. 5, 797-800 (1998)

[3] Alcock, S. G. et al., "Bimorph mirrors: The Good, the Bad, and the Ugly," Nucl. Instr. and Meth. A 710, 87-92 (2013).

[4] Alcock, S. G. et al., "Characterization of a next-generation piezo bimorph X-ray mirror for synchrotron beamlines," J. Synchrotron Rad. 22, 10-15 (2015).

[5] Sawhney, K. et al, "Characterisation of a novel super-polished bimorph mirror," 11th International Conference on Synchrotron Radiation Instrumentation - SRI 2012, Journal of Physics: Conference Series 425, 052026 (2013).

[6] Wang, H. et al., "Fast optimization of a bimorph mirror using x-ray grating interferometry," Optics Letters 39, 8 (2014).

[7] Sutter, J. P. et al., "Creating flat-top X-ray beams by applying surface profiles of alternating curvature to deformable piezo bimorph mirrors," J. Synchrotron Rad. 23, 1333-1347 (2016).

[8] Sutter, J. P. et al., "X-ray beam expansion by the application of re-entrant surface profiles to deformable bimorph mirrors,” Proc. SPIE 9965, 99650E-1 (2016).

[9] Sutter, J. P. et al., "A novel, $1 \mathrm{~m}$ long multilayer-coated piezo deformable bimorph mirror for focusing high-energy X-rays," AIP Conference Proceedings 2054, 030005 (2019).

[10] Alcock, S. G. et al., "Dynamic adaptive X-ray optics. Part I. Time-resolved optical metrology investigation of the bending behaviour of piezoelectric bimorph deformable X-ray mirrors," J. Synchrotron Rad. 26, 36-44 (2019).

[11] Alcock, S. G. et al., "Dynamic adaptive X-ray optics. Part II. High-speed piezoelectric bimorph deformable Kirkpatrick-Baez mirrors for rapid variation of the 2D size and shape of X-ray beams," J. Synchrotron Rad. 22, 10-15 (2019).

[12] Alcock, S. G. et al., "High-speed adaptive optics using bimorph deformable x-ray mirrors," Rev. Sci. Instrum. 90, 021712 (2019).

[13] Alcock, S. G., Nistea, I. and Sawhney, K., "Nano-metrology: The art of measuring X-ray mirrors with slope errors $<100$ nrad," Rev. Sci. Instrum. 87, 051902 (2016).

[14] Alcock, S. G. et al., "The Diamond-NOM: A non-contact profiler capable of characterizing optical figure error with sub-nanometre repeatability," Nucl. Instr. and Meth. A 616 2-3, 224-228 (2010)

[15] http://www.caenels.com/products/hv-adaptos/

[16] https://www.zygo.com/?/met/absolutepositionmeasurement/zps.htm

[17] de Groot, P., Deck, L. L. and Zanoni, C. A., "Interferometer system for monitoring an object," US Patents 7,636,166 (2009), 7,639,367 (2009) and 7,826,064 (2010).

[18] Deck, L. L., "High-performance multi-channel fiber-based absolute distance measuring interferometer system," Proc. SPIE 7405, 74050E (21 Aug 2009).

[19] Badami, V. G. and Abruña, E., “Absolutely: Small Sensor, Big Performance,” Mikroniek1 5-9 (2018).

[20] Sutter, J. P. et al., "Flat-top beams: Creating flat-top X-ray beams by applying surface profiles of alternating curvature to deformable piezo bimorph mirrors," J Synchrotron Rad. 23, 1333-1347 (2016). 